\title{
A transdisciplinary approach to understanding the causes of wicked problems such as the violent conflict in Rwanda
}

\author{
A VELTHUIZEN ${ }^{1}$
}

\begin{abstract}
The paper is presented against a background of many wicked problems that confront us in the world today such as violent crime, conflict that emanates from political power seeking, contests for scarce resources, the increasing reaction all over the world to the deterioration of socio-economic conditions and the devastation caused by natural disasters. This article will argue that the challenge of violent conflict requires an innovative approach to research and problem solving and proposes a research methodology that follows a transdisciplinary approach. The argument is informed by field research during 2006 on the management of knowledge in the Great Lakes region of Africa, including research on how knowledge on the 1994 genocide in Rwanda is managed. The paper will make recommendations on how transdisciplinary research is required to determine the causes of violent conflict in an African context and how practitioners and academics should engage in transdisciplinarity. It was found that transdisciplinary research is required to gain better insight into the causes of violent conflict in an African context. It requires from the researcher to recognise the many levels of reality that has to be integrated towards a synthesis to reveal new insights into the causes of violent conflict, including recognising the existence of a normative-spiritual realm that informs the epistemology of Africa. It furthermore requires a methodology that allows us to break out of the stifling constraints of systems thinking and linear processes into the inner space at the juncture where disciplines meet (the diversity of African communities).
\end{abstract}

Key Words: Africa, conflict, Rwanda, crime, genocide, violence, transdisciplinary

Disciplines: politics, education, law, epistemology, sociology, theology, management science

\section{Introduction}

Many wicked problems ${ }^{2}$ confront us in the world today. Every day we are bombarded with news of violent crime, and the less fortunate among us are witnesses or victims of violence. Wicked problems that come to mind are violent conflict that emanates from political power seeking (the protracted conflict in Libya), contests for scarce resources (the continued conflict in the Sudan), the increasing reaction all over the world to the deterioration of socio-

1 Dr Andreas Velthuizen is a senior researcher at the Institute for Dispute Resolution in Africa at the University of South Africa, Pretoria. Email: velthag@unisa.ac.za

A 'Wicked problem' is a problem that is difficult or impossible to solve because of incomplete, contradictory and changing requirements that are often difficult to recognize because of complex interdependencies that create other problems, if an effort is made to solve one aspect of a wicked problem. See Rittel, H.W.J. and Webber, M.M. 'Dilemmas in a general theory of planning', Policy Sciences, vol. 4, 1973, pp. 155-169. 
economic conditions and the devastation caused by natural disasters all over the world. Finding solutions to these problems is obviously not easy. For a start, it requires a thorough understanding of the causes, dynamics and consequences of these wicked problems.

Every challenge of violent conflict requires an innovative approach to research and problem solving. Such an approach creates a bridge between science and practice and relates to what actors should do about it in the real world. Analyzing the problem, we discover two distinct sub-problems:

- What research methodology is required to find the causes of violent conflict?

- What methodology is required to remove the causes?

The crux of this paper will be the first sub-problem (what research methodology is required to find the causes of violent conflict?). The aim of the paper is thus to propose a research methodology that follows a transdisciplinary approach. ${ }^{3}$

The researcher conducted field research during 2006 on the management of knowledge in the Great Lakes region of Africa. The field research included research on how knowledge on the 1994 genocide in Rwanda is managed. Experience of this research will serve as an empirical case study on how transdisciplinarity is required to determine the causes of violent conflict in an African context.

Firstly, the methodology followed during this field research is used to demonstrate the need for transdisciplinary research methodology, after which the discoveries of the value of transdisciplinary research will be discussed.

\section{Theoretical assumptions}

The theoretical framework for the study was the Trans-dimensional Knowledge Management Model (TDKM-M), a theoretical model that was developed as the result of research on African knowledge systems. The TDKM-M challenges existing models for conflict resolution in Africa as presented by multi-national institutions and introduces the principles of normative foundation, holistic order, collectiveness, activation of intervention and metaphysical innovation, as well as certain good practices. The TDKM-M requires the organization to participate in a collective middle ground in an equal way with communitybased groups, ensuring credible intra-action to add value to decisions and intervention actions in a multi-dimensional conflict arena, through the application of holistic knowledge production and distribution, focusing on mutual learning. (Velthuizen 2007, 311)

The model shows that knowledge can be managed to activate intervention in conflicts, replacing a culture of class-consciousness, racism and impunity with the norms of justice, truth and humaneness. Cultural change leads to the metaphysical innovation of perspectives that focus on peace in a free society, and transformation of African society into a society

Transdisciplinarity is defined as bringing together, in some fashion, distinctive components of two or more disciplines in a single mind, research endeavor, or instructional programme (Nissani 1995, 121). Transdisciplinarity is at once between the disciplines, across the different disciplines, and beyond all disciplines. The object of transdisciplinarity is to understand the present world through the unity of knowledge. (Nicolescu 2005, 2-3). 
characterized by growth, productivity and competitiveness. The model asserts that this ideal situation can be attained by the application of the principles and related good practices in the management of knowledge by both transnational organizations and African society, in an equal relationship to complement each other. The ultimate aim is to advance the African Renaissance as part of the betterment of humankind. (Velthuizen 2007, Abstract).

For the purpose of this paper, the TDKM-M is used to capture markers of successful transdisciplinary collaboration and the conditions that enable them. The TDKM-M offers the following assumptions about the nature of knowledge, notions of causality and 'inference', as well as processes of knowledge creation in Africa (Velthuizen 2007, 311).

- In the African knowledge system there is a normative-spiritual realm where political ends, strategic goals and the expectations of society are founded on the meta-norms of humaneness, peace, trust, justice and social equality (in some African societies called 'Ubuntu').

- 'Higher Minds' are part of an African knowledge system like any other knowledge system. In this system, we find thought leaders, indigenous knowledge holders, capable managers and specialist knowledge workers. These 'Higher Minds' are part of African communities of practice (COPs). They promote cross-cultural cohesion, as well as the ability to cross mental and physical boundaries into a transdisciplinary realm in a quest for synthesis of interpretation and synergy of activation.

- The African knowledge system is holistic in nature, displaying a fundamental order of global inter- or intra-activity among all entities. These entities implicate one another in a holistic bond manifesting in an intrinsically woven, balanced, inter-connected, intra-connected and iterative network of knowledge clusters managing knowledge within each other and as part of each other.

- A new fusion of transdimensional knowledge is the result of the processing of knowledge claims as knowledge flows through a network of entities, continuously renewing knowledge and using the network as social capital to improve the conditions of Africa. Protective mechanisms are flexible or may be abandoned to allow for fusion of interpretations, allowing maximum sharing while protecting own interests.

- Collectiveness that involves the harnessing of the collective memory of the village community, allowing processing of indigenous knowledge with African society participating. Collectiveness requires an atmosphere of mutual trust, respect and acceptance of the equity of all worldviews/perspectives without concern that one perspective is superior or inferior to the other.

- Activation of intervention involves transdimensional insight into the causes and effects of phenomena such as conflict that activates a synergy of interventionist initiatives by the community, civil society, government, regional organizations and global actors. Knowledge-driven actions attempt to arrest the destructive cycle of frustration that leads to violence and suffering, replacing it with the regeneration of a peaceful order, for the benefit of all humanity.

- Metaphysical innovation involving the comprehensive inner transformation of perspectives and actions of society, replaces persisting negative values such as racism and impunity with a humanizing experience, recognizing the uniqueness of humanity and the positive values of Ubuntu. 
These theoretical assumptions will be evaluated in this paper, revisiting the methodology that was used during the field research on the Genocide in Rwanda.

\section{Attempting the multi-, inter- and transdisciplinary approach}

A qualitative methodology was followed keeping in mind that knowledge of the social world includes a practical knowledge of the real world (Bourdieu 1997, 446). The study was aimed at developing an important account of how people view their world in social reality and based on developing an in-depth appreciation of structures and processes of knowledge creation.

An instinct based on many years of working in a system that forced the researcher to work and think within the boundaries of systems, prompted the researcher to approach the research on the genocide in Rwanda from a mono-disciplinary point of view; in this case, the political science point of view. Immediately the literature review took the researcher to historical causes of the conflict. The historical citations mostly tried to force the facts (and perceptions) into a model that reflects a dichotomized and dualistic view of conflict between two 'classes' or cultures without considering what the people of Rwanda had in common. ${ }^{4}$ It was found that one single discipline could not make adequate contributions to the study, due to the tendency of traditional disciplines to specialize.

The researcher had the benefit of a strategic studies background that is multi-disciplinary in nature. Therefore, the researcher could quite successfully apply political variables and considered the socio-economic, environmental, technological and legal factors that not only caused the violence in Rwanda, but also influenced the management of the outcomes.

Consequently, a multi-disciplinary approach was followed, meaning a conscious effort to examine a particular topic using different disciplinary approaches, in either the same study, or parallel to each other (Zulu \& Carroll 2007, 88). In the specific case of research into the genocide in Rwanda, the research required interaction with the legal fraternity, epistemologists, educators, sociologists, anthropologists and management scientists. The multi-disciplinary approach enabled the researcher to ask the right questions about actors and their actions; in other words, 'observable facts' that could be verified using the multiple empirical 'evidence' that was available. Research from several disciplines converges in the end to enhance understanding of knowledge in Africa. However, the multi-disciplinary nature of the research could not take the researcher out of the constraints of systems thinking. The separate treatment of disciplines did not lead to something new, and only reinforced existing paradigms about Africa.

Binary logic was not sufficient to encompass the purpose and scope of the research. Political science, and specifically sub-disciplines like strategic studies or conflict studies, proved to be too specialized and 'belong' to a community of experts, despite the multi-disciplinary nature of these fields. It became evident that disciplinary research tends to address only one level of reality. This study had to confront a complex array of realms. The more the researcher worked with the data, the more the researcher realized that political explanations, using the

4 See for instance UNESCO. 1995. Multiculturalism: a policy response to diversity. Paper prepared on the occasion of the 1995 Global Cultural Diversity Conference, 26-28 April 1995, Sydney, Australia. http://www.unesco.org/most/sydpaper.htm\#Ethnic. (Accessed 6 July 2012). 
interdisciplinary approach of historians, and the multi-disciplinary approach of security experts would not lead to any new insight that would discover some realities regarding Rwanda.

The research required an awareness of the holistic nature of the challenge far beyond explanations brought by the exponents of systems thinkers who were trying to force the conflict into a 'global system'. Such awareness would require the recognition of unseen factors that goes beyond scientific explanations. The methodology required research that would break out of the structured and limited way of conducting scientific research. The researcher had to find a research methodology that not only requires consideration of empirical practice and logical procedure, but also discards an apathetic approach to values and value systems and the tendency to dichotomize and analyze knowledge into meaningless propositions.

The researcher realized that to discover reality, critical reflection is required of the unseen factors such as values, motives and influences that lie hidden in a metaphysical dimension that the human senses are perhaps too limited to observe and analyze- another characteristic of transdisciplinarity.

Such a holistic approach had to be transdisciplinary, in the sense that it requires the researcher to:

- Transcend the boundaries of conventional academic disciplines to reflect and act in a new paradigm that goes beyond the empirically observable towards understanding of the tacit.

- Apply the methodology in the African context, addressing power disequilibrium between urban elites and the rural periphery and between Africa and the West, as well as a conscious movement away from neo-colonialism, marginalization of societies, political dogma, the selfish advancement of economic interests and contempt for indigenous cultures.

- Identify common ground where lasting solutions to wicked problems can be found.

\section{Towards a transdisciplinary approach}

'Transdisciplinarity' is not a new discipline but is a manner of seeing the world as systemic and more holistic (Max-Neef 2004, 15). All research takes place along a fluid continuum, ranging from the two imaginary poles of pure disciplinary work to a grand synthesis of all human knowledge. Knowledge contains two exemplars: research and education. Research and education can be compared by weighing four variables: the number of disciplines involved; the 'distance' between them; the novelty and creativity involved in combining the disciplinary elements and their degree of integration. (Nissani 1995, 121).

Transdisciplinary education is founded on transdisciplinary methodology, allowing us to establish links between persons, facts, images, representations, fields of knowledge and action, to discover the 'Eros of learning' during our entire life and to build permanent questioning and integration of beings. Transdisciplinarity is coherence among different levels of reality in the natural world. An immense self-consistency ('a cosmic bootstrap') seems to govern the evolution of the universe, from the infinitely small to the infinitely large, from the infinitely brief to the infinitely protracted. A flow of information is transmitted in a coherent manner from one level of Reality to another in our physical universe. (Nicolescu 2005, 7). 
What then lies between, across the disciplines, and beyond all disciplines? From the point of view of classical thought, there is absolutely nothing. The space between and beyond disciplines is empty, completely void, like the vacuum of classical physics. However, in the presence of several levels of 'reality', the space between disciplines and beyond disciplines is full, just as the quantum void is full of all potentialities such as quantum particles to the galaxies that condition the appearance of life in the universe. Nicolescu reiterates that transdisciplinary research is distinct from disciplinary research, even while being entirely complementary. Disciplinary research concerns, at most, one level of reality or fragments of reality. In contrast, transdisciplinarity concerns the dynamics engendered by the action of several levels of reality at once. The discovery of these dynamics necessarily passes through disciplinary knowledge and in the end disciplinarity, multidisciplinarity, interdisciplinarity and transdisciplinarity are like 'four arrows shot from a single bow: knowledge' towards an 'included middle'. (Nicolescu 190, 3).

As the study sought to deal with the problem of dysfunctions in contemporary society, the use of the transdisciplinary approach enabled the researcher to move beyond dichotomized and divisionary thinking into the space that lies beyond all disciplines in search of the wholeness of knowledge together with the harmony of our being and the understanding of the present universe.

\section{Collection and treatment of data in transdisciplinary research}

Transdisciplinary research within the context of African epistemology required contextualism, ${ }^{5}$ meaning access to different viewpoints available not only in diverse libraries to find local publications by African scholars, but also required participant observation, semistructured interviews and participation in focus groups of experts and specialists. In this regard, valuable data was gathered through semi-structured interviews with key informers, to find what was in the minds of the people that experienced or witnessed the events, recognising that the most valuable information could only be obtained by participating in events.

The researcher did not witness the genocide of 1994. The next best research method was to access diverse sources from different cultures who were direct observers or participants in the tragic series of events. It was found that 'truth' depicted by one or even a few disciplines cannot be universal or holistic. Views tend to be ethnocentric or biased in favour of a particular culture or civilization that attempts to establish domination over other modes of knowledge by declaring it'unscientific'. Therefore, data triangulation and recursiveness, in terms of time (historical context of the genocide), space (the broader Great Lakes region of Africa as universum) and different people, had to be applied.

In the social sciences, triangulations is often used to indicate that more than two methods are used in a study with a view to double- (or triple-) checking results. This is also called 'crossexamination'. It is an alternative to traditional criteria such as 'reliability' and 'validity'. It is a method-appropriate strategy of founding the credibility of qualitative analyses. Denzin (1978, 291) identified four basic types of triangulation:

5 Contextualism describes a collection of views that emphasize the context in which an action, utterance, or expression occurs (Price 2008, 208). In epistemology, contextualism is the treatment of the word 'knows' as context-sensitive (Stanley 2005, 16). 
- Data triangulation: involves time, space, and persons

- Investigator triangulation: involves multiple researchers in an investigation

- Theory triangulation: involves using more than one theoretical scheme in the interpretation of the phenomenon

- Methodological triangulation: involves using more than one method to gather data, such as interviews, observations, questionnaires and documents

In the case of Rwanda, transdisciplinary triangulation had to be applied, implying a research process that uses methods or tools suitable for the collection of data, irrespective to which discipline it 'belongs'. Transdisciplinary triangulation can be illustrated as follows (see Illustration 1):

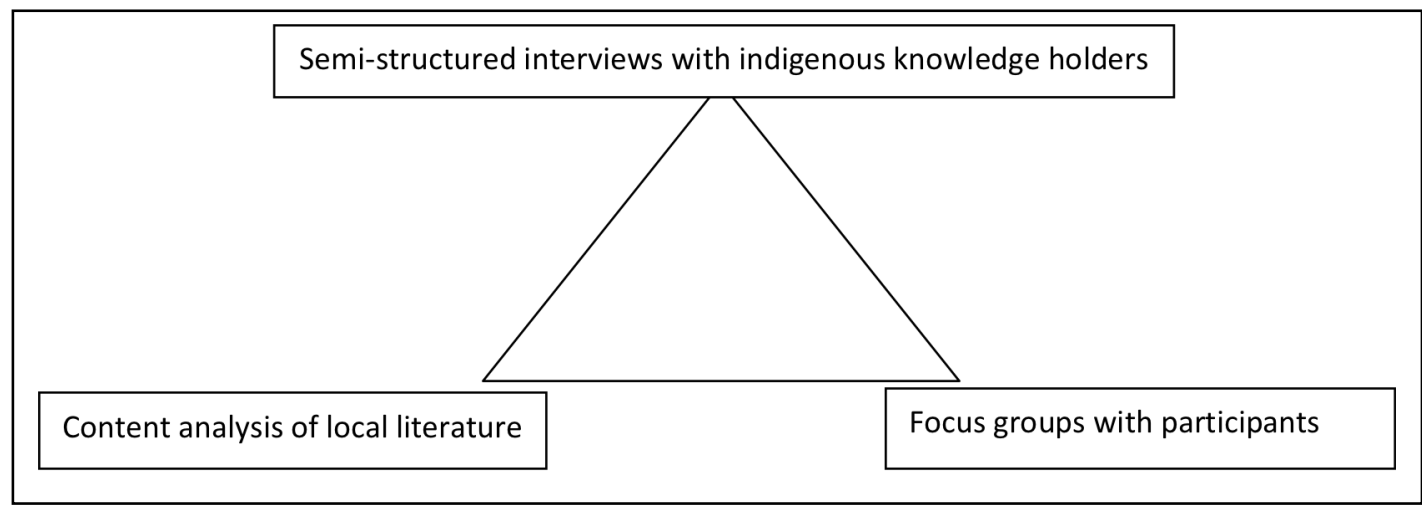

\section{Illustration 1: Transdisciplinary triangulation}

Recursiveness is a general principle of transdisciplinary research that points to the iterative procedures that characterize both the entire research process and its individual phases.

Recursiveness can be illustrated as depicted in Illustration 2.

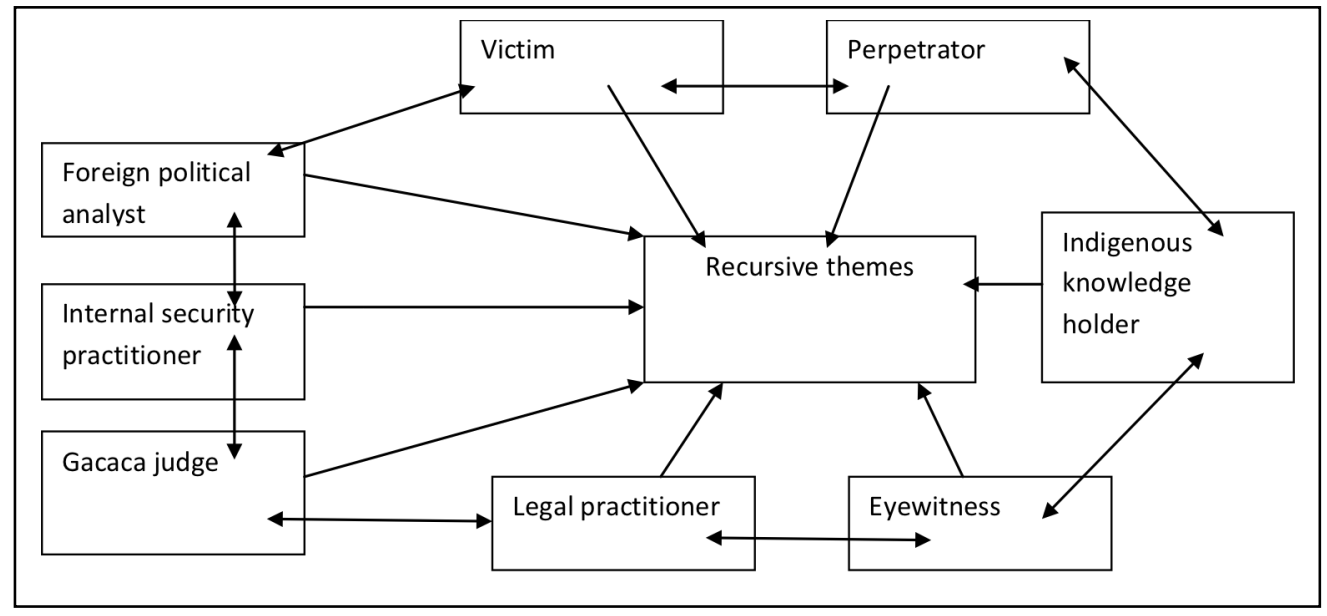

Illustration 2: Transdisciplinary recursiveness 
Recursiveness was attained when during the course of the interviews and focus groups a number of events happened that redirected the inquiry and ultimately led to a more complex data set. The initially planned structured interviews had to be handled as semi-structured, even allowed to become unstructured narrative. For instance, while the security expert described the events that led to the genocide, the judge of the Gacaca court would disagree on the details. The researcher therefore allowed deviation from a list of structured questions and invited the subjects just to tell what was on their minds. Different people from different disciplines then described their experiences and observations freely. The different sets of descriptions confirmed a disparaging and depressing picture of a context of recursive inhumane, violent actions by different perpetrators on different levels. Because of the initial handling of the data during the collection phase, it became possible to maintain the principle of recursiveness during the analysis and interpretation phase of the research process.

It was found that recursiveness requires that the research process is shaped in such a way that concepts and methods can be repeatedly tested by listening to real-world experiences that occurred in the various 'living-laboratories' of more than one discipline.

Most disciplinary research practices, interdisciplinary and transdisciplinary projects are characterized by a variety of disciplines and various practitioners in professional fields outside academia. Consequently, general evaluation methods that have been developed for disciplinary work can only be used to assess specific disciplinary contributions but are not appropriate for assessing specific inter- or transdisciplinary goals, processes and achievements. (The Swiss National Science Foundation 2011, home page).

In this study, to attain a transdisciplinary and holistic result, a certain methodology that mediates between epistemology and political science was required to access the knowledge embedded in African knowledge systems. For instance, for the field studies on Rwanda abductive logic was used for the interpretation of the data.

Abductive logic means that the mind seeks to bring facts, as modified by a new discovery, into order. The mind seeks to find a general conception; in some cases, by an act of generalization. In other cases, the mind suggests a peculiar state of facts that will 'explain' a surprising phenomenon and an already known 'law' is recognized as applicable to a suggested hypothesis. The phenomenon, under that assumption, would not be surprising but likely, or would be a necessary result. This synthesis suggesting a new conception or hypothesis is the 'abduction' (Peirce 1903, 287).

In practice, 'abduction' implies listening and observing different views expressed by different experts and actors to explain how discourse amongst people can contribute to the understanding of phenomena. It was found that abductive logic involved a special creativity to generate a new account of the way in which role-players structure reality, going beyond just reproducing meanings expressed by participants. This form of logic enabled the researcher to identify themes; patterns; trends; and specific indicators about knowledge claims and how these are managed to find the causes of violent conflict.

The evaluation, analysis and interpretation of the collected data required a holistic perspective to claim transdisciplinarity. The interpreter had to view the world through several 'windows', preferably as part of a cross-functional team that places data in a holistic context, applying experience, empathy, vision, intuition and above all, recognizing what is beyond observation to allocate meaning to data. It is therefore highly unlikely that one individual could attain 
transdisciplinarity during the research process. To follow this methodology meant the synthesizing of all forms of knowledge through discourse, in a search for understanding.

This effort has long been 'bedevilled' with aspects such as racialist myths based on outward appearances. The interdisciplinary approach may be a cure, which brings together different types of evidence in the search for the truth, implying a loose association, and no more than agreeing to common objectives. Each member of the group then works according to the techniques of own disciplines, with a meeting at the end to collate results. African culture is to give full weight to all the parts and so portray the dynamic development of the whole. In other words, the interdisciplinary method should eventually lead to a transdisciplinary approach, one in which all disciplines are represented with equal right. Life, whether individual or collective, is neither linear nor one-dimensional: it is dense, compact tissue. (Kizerbo 2000, 355-356).

Common bond links man; animal; plant; and galaxy: in the unitary principle of all reality; the self without the universe is empty'. The purpose of this re-convergence is to reveal to us that evolution and development are interchangeable and that the entire system of the interweaving of man, nature and the universe constitutes a living totality. To comprehend the new reality at which the convergence begins, we need to develop a new holistic reality, which can reveal to us the integral world that is beginning to emerge. Epistemology must go beyond scientism as developed in the West to applying the principle of the unitary structure of the universe and hence of everything consistent with the Ubuntu philosophy of 'I exist because you exist'.

Methodology to access the knowledge or wisdom of Africa has to be hermeneutical (understand how Africans see the world) because it is not only scientific reason that is involved. The predominance of 'orality' and 'verbality' requires interdisciplinarity, as one of the mechanisms to fight the rigidity of Western scientific method, but it is not enough. (Anshen 1986, xi-xii).

The predominance of orality and verbality in the African worldview presents humanity with the possibility of tracing back the origin of concepts of things and worldviews. In the pursuance of this task, interdisciplinarity is only one of the mechanisms of challenging modern 'scientific method' that focuses too narrowly on 'the disciplines', 'constructed' for production of knowledge for domination. Nabudere proposed that multi- and interdisciplinary research is not the answer to discovering the knowledge and wisdom present in African worldviews. While we may use existing MIT approaches to try to understand our reality, we do so with approaches that emanate 'from outside'. The question is whether we can move further from this external orientation 'nearer to the inside'; so that we should discover that the 'African story' knows of no disciplinary boundaries to negotiate with. As we move from the outside to the inside, we have to define new approaches of understanding that are appropriate to the African world (Nabudere 2011b, 29).

Traditional methods of acquiring knowledge and problem solving are insufficient. Transdisciplinary training is an additional method of knowledge acquisition. Universities need to innovate, evolve and adapt to be able to deal with challenges of global and long-term impact (Songca 2006, 221-230).

In terms of research, methods and methodologies, there is a greater need for a deeper search for deeper meanings of science, philosophy, law, morality, history, technology, placing all the disciplines in a trans-disciplinary frame of reference (see Illustration 3). 


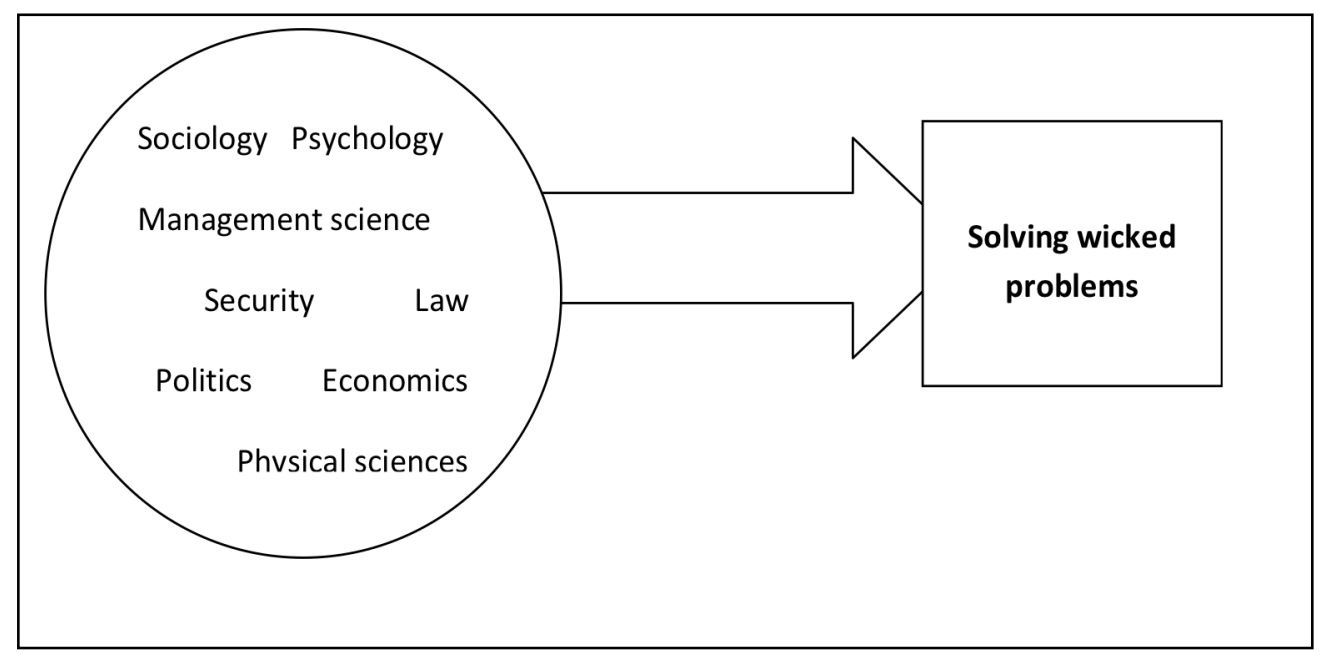

Illustration 3: Transdisciplinary research in an African epistemology

\section{Conclusions}

Trans-disciplinary research is required to determine the causes of violent conflict in an African context. Mono-disciplinarity, interdisciplinarity and multi-disciplinarity may bring forth valuable insights into a conflict, but it is when the researcher breaks out of the stifling constraints of systems thinking and linear processes set by Western hermeneutics towards critical reflection, that we develop an insight that would allow us to seek the causes of wicked challenges and engage it.

Gaining such insight and understanding into the causes of violence requires from the researcher to recognise the many levels of reality that has to be integrated towards a synthesis that reveals new insights into the causes of violent conflict, including recognising the existence of a normative-spiritual realm that informs the epistemology of Africa.

It is at the spacious 'piazza' at the juncture of many 'avenues' (or the gathering on the village lawn) that new knowledge can be created in the search for reality. It is in the living laboratory of the village community or community of practice that collective knowledge can be discovered and used to find lasting solutions by means of transdisciplinary research for the restoration of humanistic norms.

Such an intellectual adventure requires substantial courage and skills in applying the wealth of methodologies and tools that the traditional disciplines provide to erase boundaries and fuse knowledge into one transdisciplinary knowledge sphere, especially in view of the disciplinary correctness that became part of our academic world and the persistent protection of territories that comes with managerism and administrative bureaucracy. It is evident that the trap of protectionism and managerism in a constrained system does not allow for insight into wicked problems, let alone the finding of innovative and sustainable social solutions. A new holistic paradigm of interpreting phenomena is required, which would deliver a new body of knowledge that can be applied for innovation, change, transformation and the promotion of the cross-cultural cohesion of a society in conflict. 


\section{Recommendations}

It is recommended that dispute/conflict resolution practitioners engage in trans-disciplinary research by embarking on a venture to learn more about the application of transdisciplinarity as an extension of the specialist, multi- and transdisciplinary knowledge and skills they are already mastering. Such ventures will probably meet with resistance or apathy, but serious professionals are encouraged to persist in finding new knowledge on the causes of conflict, and especially a wicked problem such as genocide. Such knowledge will probably be found in the space among disciplines, where crosscutting normative and spiritual causes may reveal new perspectives on the deep-lying causes of genocide.

Academics should draw on the experience of practitioners in the business world, the public service and communities, because in most cases these practitioners are generalists and primarily transdisciplinary in their thinking. This type of collectiveness may enable academic institutions to develop new integrated and coherent transdisciplinary models on how to contribute to efforts that deal with complex and wicked problems in the real world, such as the resolution of violent conflict and other forms of disputes.

Postgraduate scholars and other knowledge workers should be encouraged to move beyond the constraints of disciplinary boundaries when deciding on research methodology. They should not allow themselves to be constrained by boundaries that were created for administrative purposes and to design protected territories where private agendas are playing out. Such an inhibition would lead to constrained thinking and a convenient escape from a reality without finding lasting solutions for the wicked problems of the world we are living in.

Leaders and managers should use transdisciplinarity towards collective sense making of a changing world to create a better society, characterized by social cohesion among entities in the spirit of honest efforts to do away with dichotomies and dualism in society, creating a new peaceful reality.

\section{References}

Anshen, R.N. 1986. Language E̋ Thought. Preface to Chomsky, N. (ed.) New York: Moyer Bell.

Bourdieu, P. 1979. A Social Critique of the Judgment of Taste. Translated by Nice, R. New York: Harvard University Press, 1984.

Denzin, N K.1978. The Research Act. $2 d$ ed. New York: McGraw-Hill.

Ki-zerbo,J. 2000. General History of Africa. Vol.1. New York: UNESCO Publishing.

Nabudere, D.W. 2011a. Towards an Afrikology of knowledge production and African regeneration. http://www.blackherbals.com/Afrikology_and_Renewal.pdf. (Accessed 9 September 2011).

Nabudere, D.W. 2011b.The global political economy and the future of Africa. Key-note address at the Mapungube Institute for Strategic Reflection (MISTRA). (Accessed 18th March 2011).

Max-Neef, M.A. 2005. 'Foundations of transdisciplinarity'. Ecological Economics. Vol. 53, no. 3. www.sciencedirect.com. (Accessed 14 October 2011). 
Nicolescu, B. 2005. Towards Transdisciplinary Education and Learning. Paper prepared for conference on 'Science and Religion: Global Perspectives', June 4-8, 2005. Philadelphia: Metanexus Institute. www.metanexus.net. (Accessed 15 November 2011).

Nissani, M. 1995. 'Fruit salads and smoothies: A working definition on interdisciplinarity'. Journal of Educational Thought. Vol.29.

Peirce, C. S. 1903. 'A Syllabus of Certain Topics of Logic'. Essential Peirce. Vol. 2. Boston: Alfred Mudge \& Son.

Price, A. W. 2008. Contextuality in Practical Reason. New York: Oxford University Press.

Rittel, H.W.J and Webber, M.M. 1973. 'Dilemmas in a general theory of planning', Policy Sciences. Vol. 4, 1973.

Songca, R. 2006. 'Transdisciplinarity: The dawn of an emerging approach to acquiring knowledge'. International Journal of African Renaissance studies. Vol.1, No. 2, 2006.

Stanley, J. 2005. Knowledge and Practical Interests. New York: Oxford University Press.

Swiss National Science Foundation. 2011. Evaluation of Inter- and Trans-disciplinary research. Experiences and reflections on best practice. TD-conference, Berne, 14-16 September 2011. http://www.transdisciplinarity.ch. (Accessed 20 September 2011).

UNESCO. 1995. Multiculturalism: a policy response to diversity.

Paper prepared on the occasion of the 1995 Global Cultural Diversity Conference, 26-28 April 1995, Sydney, Australia. http://www.unesco.org/most/sydpaper.htm\#Ethnic. (Accessed 6 July 2012).

Velthuizen, A.G. 2007. The Management of Knowledge: A Model for the African Renaissance. Doctoral Thesis. Pretoria: University of South Africa.

Zulu, I.M. and Carroll, K.K. 2007. Transdisciplinary African American Studies. Approaches and implications: A Collective Interview with James Stewart, November 26, 2007. http://www.jpanafrican.com/docs/vol2no2/TransdisciplinaryAfricanAmericanStudies ApproachesAndImplications.pdf. (Accessed 6 July 2012) 